\title{
Simulated Radio Images and Light Curves of SN 1993J
}

\author{
Vikram V. Dwarkadas $^{1}$, Amy J. Mioduszewski ${ }^{2}$, and Lewis T. Ball ${ }^{3}$
}

${ }^{1}$ ASCI FLASH Center, Univ of Chicago, 5640 S. Ellis Ave, Chicago IL 60637 vikram@flash .uchicago .edu

${ }^{2}$ NRAO, P.O. Box O, Socorro, NM 87801, USA; amiodusz@nrao.edu

${ }^{3}$ CSIRO ATNF Parkes Observatory, P.O. Box 276, Parkes NSW 2870, Australia lewis.ball@csiro.au

Summary. We present calculations of the radio images and light curves from supernovae, based on high-resolution numerical simulations of the hydrodynamics and radiation transfer in a spherically symmetric medium. As a specific example we model the emission from SN1993J. This supernova does not appear to be expanding in a self-similar fashion, and cannot be adequately fitted with the often-used analytic mini-shell model. We present a good fit to the radio evolution at a single frequency. Both free-free absorption and synchrotron self-absorption are needed to fit the light curve at early times, and a circumstellar density profile of $\rho \sim r^{-1.7}$ provides the best fit to the later data. Comparisons of VLBI images of SN1993J with synthetic model images suggest that internal free-free absorption completely obscures emission at $8.4 \mathrm{GHz}$ passing through the center of the supernova for the first few tens of years after explosion.

\section{Introduction}

Radio Supernova (RSN) light curves are characterized by an initial rapid increase of radio flux to maximum, followed by a power-law decrease with time. High brightness temperature indicates a non-thermal origin for the emission, and it is now accepted that the emission is synchrotron in origin, due to the spiraling of relativistic electrons in a magnetic field. The wavelengthdependent turn-on suggests that the initial rise in the light curve is due to decreasing absorption as the shock expands in an optically thick medium.

In the past, the computation of SN radio light curves has been accomplished mainly via semi-empirical methods, primarily the mini-shell model developed by Chevalier [3] and extended by Weiler et al. [9]. These empirical methods assume a self-similar evolution for the SN shock front, with the shock radius expanding as a power law in time $\mathrm{r}_{s} \propto t^{m}$, where $m$ is a constant [3]. The energy distribution of the relativistic electrons is assumed to be a power law of constant spectral index, and the energy density of the relativistic electrons and that of the magnetic field are assumed to each be a constant fraction of the thermal energy density behind the expanding supernova shock. 


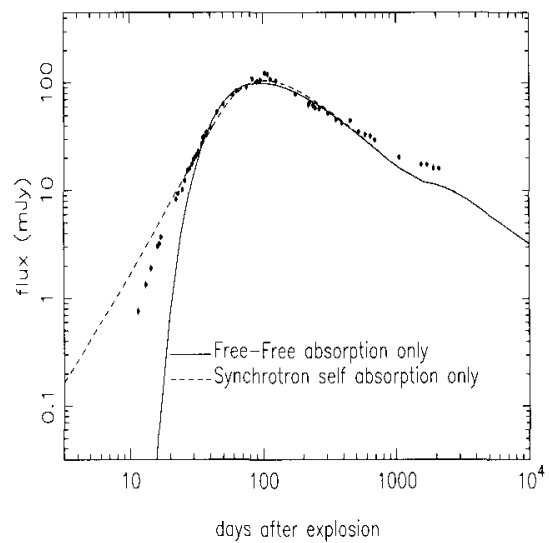

Fig 1a

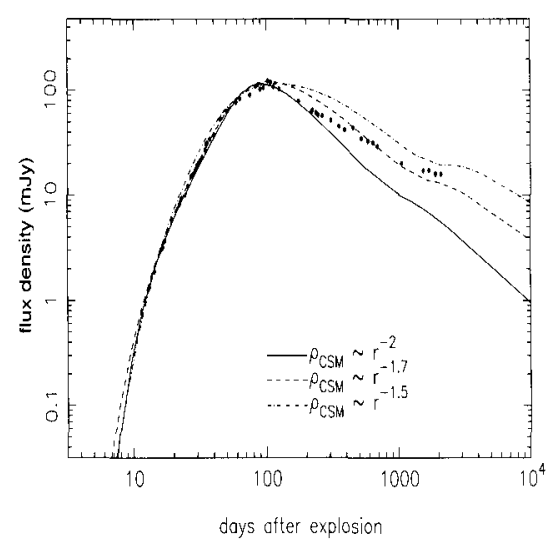

Fig $1 b$

In this article we outline a more robust and general technique for computing the radio light curves of young SNe. Details of our method are outlined in Mioduszewski et al. [5]. Herein we briefly describe the technique and the major results for the well-studied SN 1993J, as well as update the results with comparisons to more recent data.

\section{Methods and Techniques}

- We start with a computation of the hydrodynamic evolution of the SN remnant, calculated using VH-1, a 3-dimensional, finite-difference, high resolution, shock-capturing code based on the Piecewise Parabolic Method. This step immediately distinguishes our method from the minishell model, since we do not need to assume a self-similar expansion for the SN shock. Our technique is applied to SN 1993J, where the observations indicate that the expansion is not self-similar.

- The light curves are produced by calculating the transfer of radiation along a line-of-sight through the SN.

- We assume that the injection of relativistic particles at the shock follows a power-law, $N(E) \propto E^{-\gamma}$.

- We start with spherically symmetric simulations. These give pressure, density and temperature at each grid point and every time step.

- The code takes the simulations and rotates them to form a 3D sphere embedded in a Cartesian grid.

- The synchrotron emission and absorption is computed along each ray. If $N(E)=K E^{-\gamma}$, then emissivity $j$ and opacity $\kappa$ are given by

$$
j \sim K B^{(\gamma+1) / 2} \nu^{-(\gamma-1) / 2}
$$




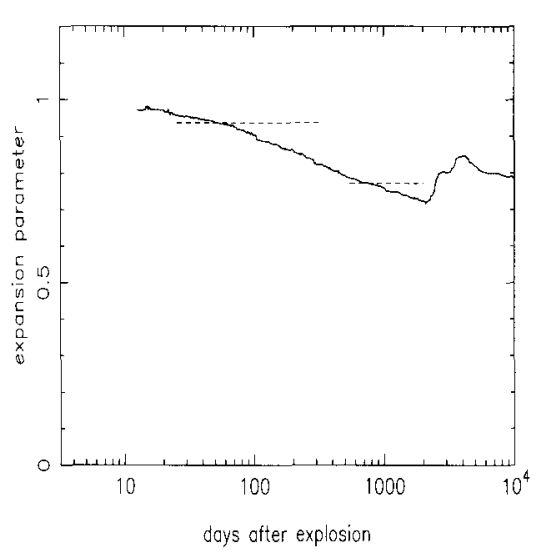

Fig $2 \mathrm{a}$

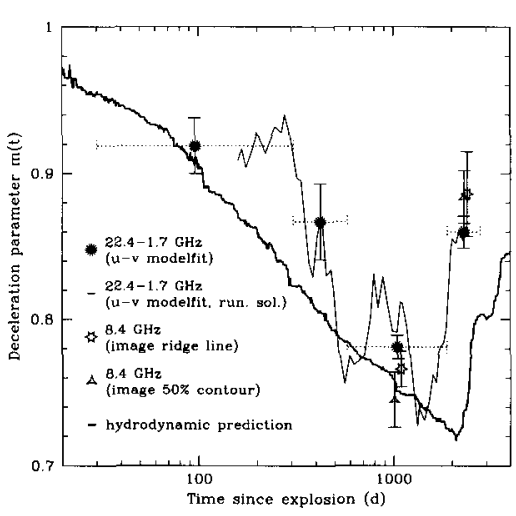

Fig $2 b$

$$
\kappa \sim K B^{(\gamma+2) / 2} \nu^{-(\gamma+4) / 2} .
$$

- In this work we assume that both magnetic energy density $u_{B}$ and relativistic particle energy density $u_{r e l}$ are proportional to the thermal energy density.

$$
u_{B}=\xi_{B} u_{t h}, \quad u_{r e l}=\xi_{\text {rel }} u_{t h}
$$

- We take into account the external free-free absorption, which depends on the temperature and density profile of the ambient medium.

- The emissivity and absorption are used to calculate the optical depth $\tau$, and finally the intensity $I_{\nu}(r)$. Integration is carried out using the trapezoidal rule.

- The result is a 2D array of surface brightness, which is used to make an image of the source and calculate the total flux.

\section{SN1993J}

As a first example we focus on SN 1993J - one of the brightest and best studied $\mathrm{SNe}$ in the Northern Hemisphere. The radio flux evolution of SN $1993 \mathrm{~J}$ has been followed in detail by Bartel et al. [1,2] and Pérez-Torres et al. $[6,7]$ using VLBI.

If shock radius $R_{s} \propto t^{m}$, then $m$ is called expansion parameter. For powerlaw models, $m$ is constant with time. But for SN 1993J, $m$ decreases with time $[1,2]$. Therefore the evolution is NOT self-similar, which perhaps implies that power-law density ejecta are not a reasonable assumption. For the ejecta structure we have therefore used Model $4 \mathrm{H} 47$ of Suzuki \& Nomoto [8]. In this 


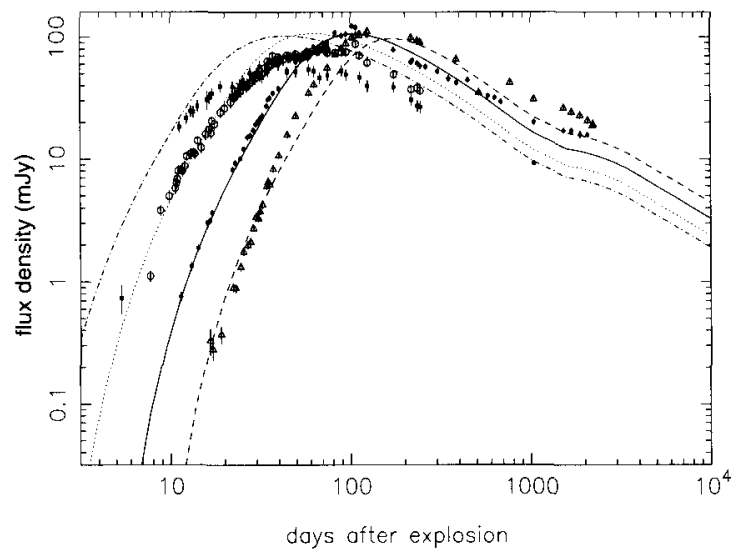

Fig 3

case the ejecta density shows considerably more structure than the commonly used "power-law" models.

In order to fit the X-ray and optical light curves, Nomoto et al. find that the CSM density profile must decrease more slowly than $r^{-2}$, which would be the case for a steady wind. Fransson and Bjornsson [4], however find that an $r^{-2}$ profile is adequate to fit the radio light curve. Therefore we have tried 3 CSM density profiles, with density decreasing as $r^{-1.7}, r^{-1.5}$ and $r^{-2 .}$.

Free-free absorption (FFA) alone results in an exponential rise of the light curve while synchrotron self absorption (SSA) alone results in a power-law increase (Fig. 1a). In order to fit the light curve we find that both FFA and SSA must be included, and that a CSM density profile of $r^{-1.7}$ provides the best fit to the observed light curve at $8.4 \mathrm{GHz}$ (Fig. 1b). The data is represented by dots in the figure.

As mentioned above, the expansion parameter $m$ (where $\mathrm{R}_{s} \propto t^{m}$ ) is not constant for SN 1993J, implying a non-self-similar evolution. Fig. 2a shows the evolution of the expansion parameter with time from our simulation of the interaction of a $\mathrm{SN}$ with an ejecta density profile described by the $4 \mathrm{H} 47$ model evolving in a medium whose density varies with radius as $r^{-1.7}$. The dashed lines in the figure are error bars for the expansion parameter measured by Bartel et al. [2]. Our simulations are broadly consistent with these observations.

The model $4 \mathrm{H} 47$ exhibits local density maxima in the ejecta density profile. Impact of the reverse shock with one of these local density maxima results in a sudden increase in the expansion parameter around day 2300 (see Fig. 2a). Observations by Bartel et al. [2] show a similar rise in the expansion parameter (Fig. 2b), albeit somewhat earlier. It is possible that the change in the observed deceleration is due to a change in the CSM density or some other cause unrelated to the ejecta structure, but the coincidence is 


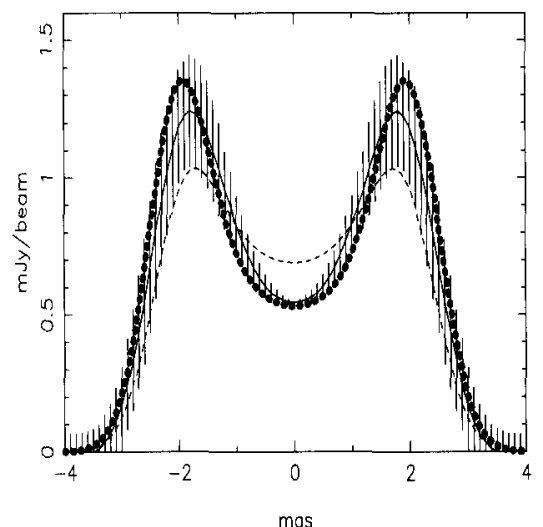

Fig $4 \mathrm{a}$

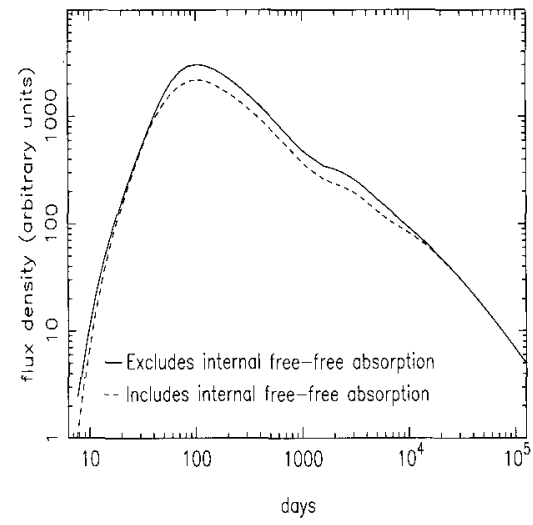

Fig $4 b$

striking nevertheless. It is also possible that in a more realistic multidimensional simulation any local density maxima would be unstable and would quickly smooth out.

\subsection{SN1993J Light Curves}

Our technique is to fit the observed light curve at one fiducial frequency 8.4 GHz. The frequency dependence at all epochs is simply $\nu^{-\alpha}$ (where $\alpha=0.55$ is the spectral index determined by this best fit model of the light curve), and so the light curve at any other frequency has the same shape as that at the fiducial frequency. The observed flux densities from SN1993J and the corresponding model light curves are shown in Fig. 3. The different frequencies indicated are: $22.5 \mathrm{GHz}$ (filled boxes, dot-dashed line), $15.0 \mathrm{GHz}$ (open circles, dotted line), $8.4 \mathrm{GHz}$ (filled circles, solid line), and $4.9 \mathrm{GHz}$ (open triangles, dashed line)

\subsection{Internal Absorption}

In the early stages, the internal free-absorption was found to be important in order to match the surface brightness profile obtained from our simulated images to that observed. Fig. 4a shows the azimuthally averaged profile of the observed $8.4 \mathrm{GHz}$ emission from SN 1993J on day 1349 (solid line with error bars), compared to that obtained from our simulations, excluding (dashed line) and including (dotted line) internal free-free absorption, convolved with the same beam as the image. The improvement in the fit when the internal free-free absorption is taken into account is readily apparent. Fig. 4b shows the change in the light curve when the internal absorption is taken into account. Our model suggests that the optical depth at $1 \mathrm{GHz}$ does not fall to 
unity until around 150 years after the explosion. This suggests that a central radio pulsar would be undetectable for many tens of years at frequencies below $1 \mathrm{GHz}$, although multidimensional effects, especially instabilities, that have not been included here may allow the radiation to escape somewhat earlier. Further details can be obtained from Mioduszewski et al. [5].

Acknowledgement. VD is supported by the US. Department of Energy grant number B341495 to the ASCI Flash Center (U Chicago), and by Award \# AST-0319261 from the National Science Foundation.

\section{References}

1. N. Bartel et al. : Science 287, 112 (2000)

2. N. Bartel et al. : Astrophys. J. 581, 404 (2002)

3. R.A. Chevalier: Astrophys. J. 258, 7901982

4. C. Fransson, C.-I. Bjornsson: Astrophys. J. 509, 861 (1998)

5. A.J. Mioduszewski, V.V. Dwarkadas, L. Ball: Astrophys. J. 562, 869 (2001)

6. M.A. Perez-Torres, A. Alberdi, J.M. Marcaide: Astron. Astrophys. 394, $71(2002)$

7. M.A. Perez-Torres, A. Alberdi, J.M. Marcaide: Astron. Astrophys. 374, 997 (2001)

8. T. Suzuki, K. Nomoto: Astrophys. J. 455, 658 (1995)

9. K.W. Weiler, R.A. Sramek, N. Panagia: In: IAU Colloq. 145, Supernovae and Supernova Remnants, ed. by R. McCray, Z. Wang (Cambridge: Cambridge Univ. Press, 1996) p. 283 